\title{
MORBIDADE PSÍQUICA ENTRE TRABALHADORES DE FEIRA DE SANTANA, BAHIA, SEGUNDO GÊNERO
}

\author{
$\underline{\text { Ludmilla Couto da Silva1; }}$;ânia Maria de Araújo² e Técia Maria Santos Carneiro e \\ Cordeiro $^{3}$ \\ 1. Bolsista PIBIC/CNPq, Graduanda de Psicologia, Universidade Estadual de Feira de Santana, e-mail: scmila8@ gmail.com \\ 2. Orientadora, Coordenadora do Núcleo de Epidemiologia, Departamento de Saúde, Universidade Estadual de Feira de \\ Santana, e-mail: araujo.tania@uefs.br \\ 3. Pesquisadora do Núcleo de Epidemiologia, Departamento de Saúde, Universidade Estadual de Feira de Santana, e-mail: \\ teciamarya@yahoo.com.br
}

PALAVRAS-CHAVE: morbidade psíquica; trabalho; gênero

\section{INTRODUÇÃO}

A morbidade psíquica, cujos efeitos reverberam na saúde e na qualidade de vida dos indivíduos, pode ter como nexo causal, variados aspectos presentes na experiência humana, com destaque para os fatores relacionados à esfera laboral dos mesmos.

A precarização da estrutura, da política e das relações sociais que permeiam o mundo organizacional constitui um risco ao desenvolvimento de transtornos mentais, visto que estes se apresentam de modo incompatível com a capacidade biológica e fisiológica do trabalhador (Franco, Druck, Seligmann-Silva, 2010). Pode-se acrescentar a este fato, as distinções de gênero que fazem parte da configuração social no mundo do trabalho, as quais têm definido de modo significativo a permanência no emprego e as condições do mesmo (OIT, 2010) produzindo, por conseguinte, adoecimentos psíquicos de modo diferenciado em homens e mulheres.

Nessa perspectiva, o objetivo geral do presente estudo foi avaliar a morbidade psíquica entre trabalhadores de Feira de Santana, Bahia, segundo gênero. Tendo em vista que os transtornos mentais refletem na qualidade de vida dos indivíduos e em várias dimensões da vida dos acometidos, os dados estatísticos advindos dessa pesquisa contribuirão na reflexão crítica acerca das variáveis e das desigualdades sociais envolvidas nesse processo, viabilizando o desenvolvimento de políticas públicas que estimulem a efetiva igualdade de oportunidades no mercado de trabalho e de tratamento aos indivíduos, independente de gênero.

\section{METODOLOGIA}

Este estudo faz parte da Pesquisa intitulada 'Caracterização da Saúde Mental de Feira de Santana, Bahia, Brasil" desenvolvido pelo Núcleo de Epidemiologia (NEPI) da Universidade Estadual de Feira de Santana (UEFS). Trata-se de um estudo epidemiológico de corte transversal de caráter exploratório analítico.

Neste estudo foram estudados os trabalhadores residentes da zona urbana de Feira de Santana, Bahia, segundo gênero. Os dados foram coletados mediante a aplicação de um questionário, composto por blocos de questões (sociodemográficas, ocupacionais, de hábitos de vida, condições de saúde e aspectos psicossociais do trabalho). Para mensurar as prevalências dos desfechos - depressão, ansiedade, transtorno de pânico, transtorno de compulsão alimentar e bulimia nervosa - foi utilizado o Patient Health Questionnaire (PHQ) (SANTOS et al., 2013).

Assim, com base neste instrumento as estimativas das prevalências destas cinco morbidades psíquicas foram analisadas de modo a verificar as diferenças nas frequências desses efeitos sobre a saúde mental segundo gênero. E também foi analisada a distribuição 
das prevalências dos desfechos de interesse segundo características sociodemográficas (sexo, idade, escolaridade, situação conjugal, raça/cor, renda mensal).

O processamento dos dados foi realizado pelo programa estatístico "StatisticalPackage for the Social Sciences" (SPSS) for Windows, versão 15.0. e OpenEpi versão 3.03a. Foram calculadas as medidas de associação pertinentes, razão de prevalência e seus respectivos Intervalos de Confiança (IC) de $95 \%$ e obtidos os valores de p através do teste qui-quadrado, sendo que, para aqueles valores esperados $<5$, foi utilizado o teste Exato de Fisher, assumindo-se, para significância estatística, $\mathrm{p} \leq 0,05$.

Os protocolos de pesquisa, ao qual este estudo integra, foram avaliados e aprovados pelo Comitê da Ética em Pesquisa com Seres Humanos da Universidade Estadual de Feira de Santana sob parecer n ${ }^{\circ} 042 / 06$.

\section{RESULTADOS E DISCUSSÃO}

No estudo houve 1.557 participantes, destes $54,7 \%$ eram do sexo feminino "trabalhadoras" e 45,3\% do sexo masculino "trabalhadores". Em relação às características sociodemográficas, o estudo revelou maior percentual de trabalhadoras na faixa etária de 31 anos a 45 anos (39,5\%), sem companheiro (a) (50,9\%), sem ensino superior $(94,5 \%)$, que autorreferiram raça/cor da pele negra $(85 \%)$, renda mensal de até um salário mínimo $(59,7 \%)$, com vínculo de trabalho informal $(73,6 \%)$ e sobrecarga doméstica alta $(47,5 \%)$.

No que se refere aos trabalhadores, a maioria estava na faixa etária de 30 anos ou menos (38,9\%), com companheiro (a) $(60,1 \%)$, sem ensino superior $(97,4 \%)$, que autorreferiram raça/cor da pele negra $(85 \%)$, renda mensal de mais de um salário mínimo (70,4\%), com vínculo de trabalho informal $(65,4 \%)$ e sobrecarga doméstica baixa $(61,3 \%)$.

No tocante a características ocupacionais, a maior parte das trabalhadoras tinha mais de 5 anos exercendo atividade laboral (54,3\%). Quanto ao turno de trabalho, houve predomínio de mulheres que trabalhavam durante o dia $(76,1 \%)$, com carga horária de trabalho semanal de 40 horas ou menos $(59,1 \%)$, que trabalhavam de 1 a 5 dias na semana $(52,4 \%)$ e que não possuíam outra atividade que lhe oferecesse rendimento $(85,4 \%)$. Houve predomínio de trabalhadores que tinham mais de 5 anos inseridos no mercado de trabalho $(56,2 \%)$, em escala alternada $(23,1 \%)$, em mais de 5 dias da semana $(55,7 \%)$, com carga horária semanal de mais de 40 horas $(56,8 \%)$ e que não possuíam outra atividade laboral remunerada $(87,7 \%)$.

No que diz respeito às características psicossociais do trabalho de acordo com o modelo demanda-controle, registra-se que as atividades laborais exercidas pelas trabalhadoras eram caracterizadas em maior proporção pela baixa demanda psicológica $(54,4 \%)$ e pelo baixo controle $(60,1 \%)$. Observou-se que havia maior proporção de mulheres em situação de trabalho passivo $(30,6 \%)$. Do mesmo modo, os trabalhadores ocupavam empregos caracterizados por baixa demanda psicológica $(52,4 \%)$ e pelo baixo controle $(55,1 \%)$. Todavia, entre os mesmos, verifica-se que o maior percentual de homens estava em situação de alta exigência no trabalho $(27,8 \%)$.

Quanto aos hábitos de vida, a maior parte dos trabalhadores, tanto do gênero feminino $(87,2 \%)$, quanto do gênero masculino $(94,2 \%)$, praticavam atividade de lazer. Com relação ao consumo de álcool $26,4 \%$ das mulheres e $55 \%$ dos homens relataram hábito de consumir bebida alcoólica. No que se refere ao uso de tabaco, notou-se a maior proporção das trabalhadoras $(90,2 \%)$ e dos trabalhadores $(82,2 \%)$ de não fumantes.

A prevalência de transtornos de ansiedade entre as trabalhadoras foi de $4,9 \%$ e de $2,1 \%$ entre os trabalhadores. Entre as trabalhadoras, houve associação negativa estaticamente significante com a idade de 30 anos ou menos $(\mathrm{RP}=0,43$; valor de $\mathrm{p}=0,05)$. Entre os trabalhadores, não houve associações estatísticas significantes (Figura 1). 
A prevalência de transtorno depressivo segundo a gravidade entre as trabalhadoras foi de $22,3 \%$ para quem apresentou depressão leve, 7,3\% moderada, 3,7\% moderada severa e $1,1 \%$ severa e entre os trabalhadores foi de $14,2 \%$ para depressão leve, $4,8 \%$ moderada, $1,3 \%$ moderada severa e $0,3 \%$ severa. Entre as trabalhadoras, a associação positiva foi estaticamente significante apenas em ter renda de até 1 salário mínimo: depressão leve ( $\mathrm{RP}=$ $1,54$; valor de $\mathrm{p}<0,01)$, depressão moderada ( $\mathrm{RP}=2,42$; valor de $\mathrm{p}<0,01)$ e depressão moderada severa $(R P=2,80$; valor de $\mathrm{p}=0,01)$. E houve associação negativa estaticamente significante para aquelas que não têm ensino superior: depressão leve $(\mathrm{RP}=0,32$; valor de $\mathrm{p}<0,01$ ) (Figura 1).

Para os trabalhadores, a associação positiva foi estaticamente significante em ter renda de até 1 salário mínimo: depressão leve $(\mathrm{RP}=1,56$; valor de $\mathrm{p}=0,01)$ e depressão moderada $(\mathrm{RP}=2,91$; valor de $\mathrm{p}<0,01)$ (Figura 1).

A prevalência de transtorno de pânico entre as trabalhadoras foi de 5,9\% e de 2,8\% entre os trabalhadores. Entre as trabalhadoras, não houve associações estatísticas significantes. Entre os trabalhadores, a associação positiva foi estaticamente significante apenas para aqueles que tinham de 31 a 45 anos de idade $(\mathrm{RP}=8,11$; valor de $\mathrm{p}=0,02)$ (Figura 1).

A prevalência de transtorno de compulsão alimentar foi de $5,1 \%$ entre trabalhadoras e 3,3\% entre os trabalhadores. Entre as trabalhadoras e trabalhadores, a associação positiva foi estaticamente significante para aqueles que tinham 30 anos ou menos de idade $(\mathrm{RP}=3,15$; valor de $\mathrm{p}=0,03),(\mathrm{RP}=5,00$; valor de $\mathrm{p}=0,02)$. A prevalência de bulimia foi de $0,8 \%$ entre trabalhadoras e $1,0 \%$ entre os trabalhadores. Todavia, não houve associação estaticamente significante (Figura 1).
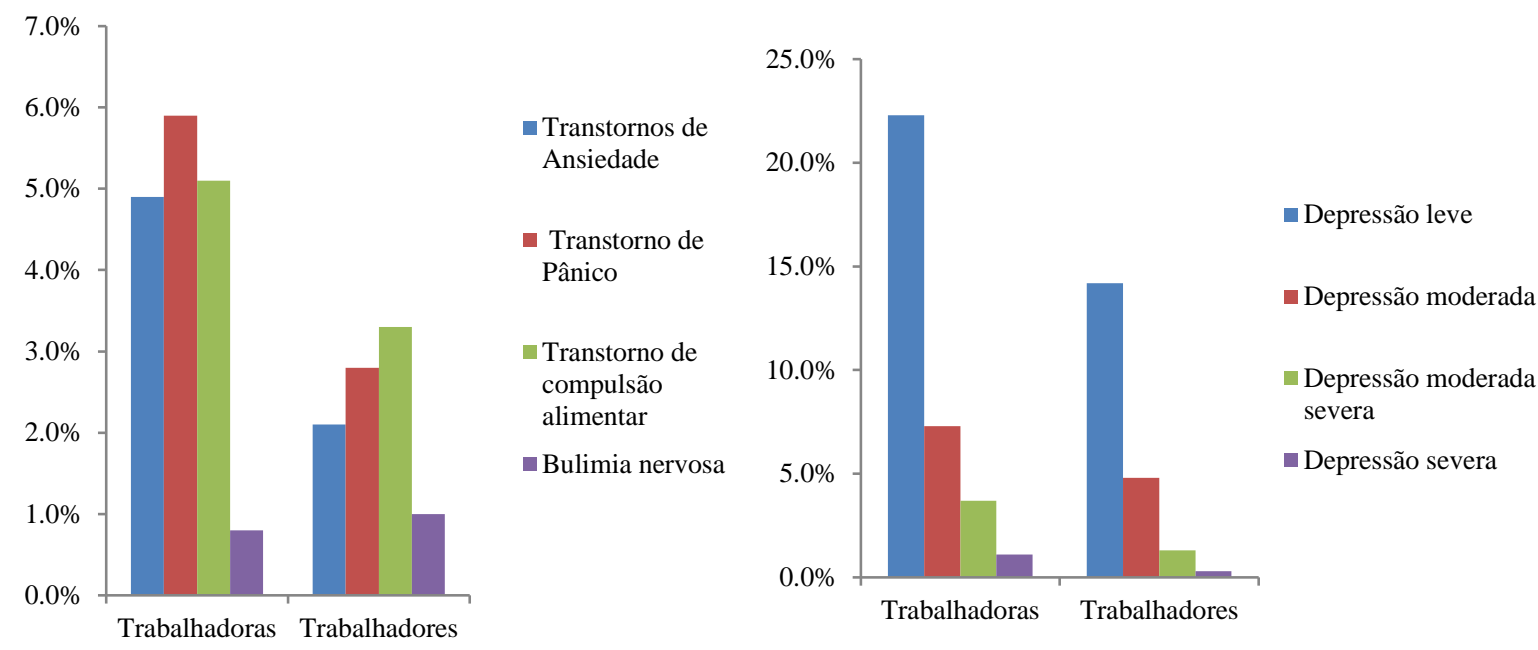

Figura 1. Prevalência de morbidades psíquicas entre trabalhadores de Feira de Santana, Bahia, segundo gênero.

Lima (1999) por meio dos dados de estudos epidemiológicos da depressão realizado nas últimas décadas verificou, no que diz respeito à situação conjugal, que a depressão pode se desenvolver mais frequentemente entre mulheres divorciadas ou separadas, do que entre as casadas, confirmando os dados do presente estudo.

Conforme Terra (2010), o status social é caracterizado em função do nível salarial. Conjectura-se que a baixa remuneração, enquanto condição precária do trabalho pode ser o responsável pela insatisfação com a atividade laboral que se realiza, o que resulta em esgotamento mental e provável adoecimento, a exemplo, de quadros depressivos. 
Os jovens vivenciam de forma mais intensa obstáculos na busca por emprego e, mesmo quando inseridos no mercado de trabalho, estão propensos as mais degradantes condições laborais (MINISTÉRIO DO TRABALHO E EMPREGO, 2010), ainda que trabalhando mediante vínculo formal. Tais circunstâncias organizacionais refletem negativamente na saúde mental dos mesmos, podendo provocar o desenvolvimento de transtornos de ansiedade e de compulsão alimentar, como apresentado neste estudo.

Trabalhadoras que possuem elevado grau de escolaridade terão mais chances e oportunidades de conquistar atividades laborais de maior prestígio social, melhores remunerações e condições de trabalho. Nesse sentido, no caso de mulheres que não possuam ensino superior, essa realidade se inverte, já que estará submetida a piores condições laborais, precarizadas relações de trabalho, marcadas pela informalidade e ausência de garantias sociais (Gonzalez, 2011), o que poderá ocasionar quadros de ansiedade e depressão.

\section{CONSIDERAÇÕES FINAIS}

Conclui-se que a distribuição desigual das principais morbidades psíquicas segundo gênero expressa que os determinantes sociais, econômicos e laborais relacionados a saúde mental são diferentes entre homens e mulheres (Pinho, Araújo, 2012). As trabalhadoras se apresentaram mais propensas a desenvolver transtornos de ansiedade, transtorno de compulsão alimentar e depressivo do que os trabalhadores, revelando que apesar dos espaços conquistados, ainda não se vivencia uma realidade de igualdade entre os gêneros.

Nesse sentido, analisar os fatores associados ao adoecimento mental das trabalhadoras é essencial para se planejar políticas públicas que transformem a realidade de precariedade laboral, a qual ainda está sujeitas às mulheres, estimulando a efetiva igualdade de condições e oportunidades no mercado de trabalho entre mulheres e homens.

\section{REFERENCIAS}

CARTILHA AGENDA NACIONAL PARA O TRABALHO DOCENTE. MINISTÉRIO DO TRABALHO E EMPREGO, 2010.

FRANCO, T.; DRUCK, G.; SELIGMANN-SILVA, E. 2010. As novas relações de trabalho, o desgaste mental do trabalhador e os transtornos mentais no trabalho precarizado. Rev. bras. Saúde ocup. 35(122): 229-248.

GONZALEZ, D.F. 2011. Políticas laborais de igualdade de gênero: o Programa Pró-Equidade de Gênero no Brasil. In: II Simpósio Gênero e Políticas Públicas, Londrina, p.1-12.

LIMA, M. S. 1999. Epidemiologia e impacto social. Rev. Bras. Psiquiatr. 21(1): 01-05.

ORGANIZAÇÃO INTERNACIONAL DO TRABALHO (OIT). 2010. Igualdade de gênero e raça no trabalho: avanços e desafios. Brasília, 216p.

PINHO, P. S.; ARAUJO, T. M. 2012. Associação entre sobrecarga doméstica e transtornos mentais comuns em mulheres. Rev. bras. Epidemiol. 15(3): 560-572.

TERRA, F.S. 2010. Avaliação da ansiedade, depressão e autoestima em docentes de Enfermagem de universidade pública e privada. Universidade de São Paulo, Tese. 